\title{
Zur Problematik der Rekontextualisierung spätzeitlicher ägyptischer Bronzestatuetten
}

\begin{abstract}
Weiß, Katja: Ägyptische Tier- und Götterbronzen aus Unterägypten. Untersuchungen zu Typus, Ikonographie und Funktion sowie der Bedeutung innerhalb der Kulturkontakte zu Griechenland. Wiesbaden: Harrassowitz 2012. XXXVI, 1171 S., 2 Teile, 7 Abb., 79 Taf., 145 Tab., 1 Kart. $4^{\circ}=$ Ägypten und Altes Testament 81. Hartbd. $€ 198,00$. ISBN 978-3-447-06719-5.
\end{abstract}

Besprochen von Eva Lange-Athinodorou: Würzburg/Deutschland, E-Mail: eva.lange@uni-wuerzburg.de

DOI 10.1515/olzg-2017-0001

Das zu besprechende Werk geht auf eine 2008/09 an der Universität Mainz eingereichte Dissertationsschrift zurück. Seine Grundlage bildet die Bearbeitung einer umfangreichen Klasse der materiellen Kultur des spätzeitlichen Ägyptens, der Bronzestatuetten von Götter- und Tiergestalten aus Unterägypten. Damit fügt es sich in den Umkreis objektkonzentrierter Forschungsarbeiten, wie sie beispielsweise die zweibändige Publikation von Günther Roeder zu den Bronzefiguren des Berliner Ägyptischen Museums von $1956^{1}$ und die materialreiche Abhandlung zu ägyptischen Bronze- und Goldstatuetten von J. F. Aubert und L. Aubert, $2001^{2}$, darstellen. Wie die Autorin selbst feststellen muss, hat jedoch bisher keine umfangreiche und methodisch angelegte Auseinandersetzung mit dieser Thematik stattgefunden, was eine Neubearbeitung des Themas lohnenswert erscheinen lässt.

Tatsächlich ist dieser Mangel angesichts der großen Menge an Bronzestatuen in den ägyptischen Sammlungen und ihrer Präsenz in Ausstellungen verschiedenster Thematiken bemerkenswert. In der Forschung werden Bronzestatuetten zumeist hinsichtlich ihrer Aussagekraft bezüglich bestimmter Götterikonographien und eines möglichen Bezugs zu lokalen Tempeln und Kulten ausgewertet, ohne dass weitere Bedeutungsebenen wie $\mathrm{z}$ B. die komplexen Prozesse des Votivwesens, die Identitäten der Stifter usw. untersucht würden. Auch das oben genannte Werk von F. Aubert und L. Aubert konzentriert sich vor-

1 G. Roeder, Mitteilungen aus der ägyptischen Sammlung. Band VI. Ägyptische Bronzefiguren, Berlin, 1956.

2 J. F. Aubert, L. Aubert Bronzes et or égyptiens, Contributions à l'Égyptologie 11, Paris, 2001. rangig auf die Rolle von Bronzestatuetten als Repräsentanten der verschiedenen ägyptischen Götterkreise.

Der erste Band der Untersuchung von Katja Weiß, der den Textteil enthält, beginnt mit einer Einleitung, in der die Autorin einen sehr knappen Forschungsüberblick gibt und die ihrer Arbeit zugrundeliegende Begrenzung auf die Götter- und Tierbronzen aus Fundorten Unterägyptens mit ihrer eindeutigen Rekontextualisierbarkeit in die hier interessierende, ausschließlich „religiöse Sphäre“ (im Gegensatz zu Menschendarstellungen und anthropomorphen oder tiergestaltigen Gebrauchsgegenständen aus Bronze: S. 2) erläutert. Auch wenn dieses Auswahlkriterium (Götterfiguren vs. Gebrauchsgegenstände) zunächst einleuchtet, ist sich die Rezensentin hier jedoch unsicher, inwieweit sie einer so apodiktisch formulierten scharfen Trennung zwischen Alltagswelt und Religion resp. Kult tatsächlich folgen kann. Die Konzentration auf Fundorte Unterägyptens begründet die Autorin dann mit dem Verweis auf die hier befindlichen lokalen Machtzentren in der Dritten Zwischenzeit und Spätzeit, in denen sich ein reiches Bronzehandwerk entfaltete. Tatsächlich spiegelt sich dies ja in der Vielzahl von Bronzestatuetten mit Herkunftsangaben aus unterägyptischen Stätten wieder. Dabei und im Folgenden begeht aber die Autorin den Fehler, Herkunftsangabe und Fundort unkritisch gleichzusetzen, was später im Detail durchaus Schwierigkeiten bereitet (s.u.). Hinzu kommt, dass den nicht selten problematischen Herkunftsangaben der zum Großteil aus Sammlungen stammenden Objekte, die die Materialbasis der Studie ausmachen, a priori sehr vertrauensvoll begegnet wird. Die Rezensentin ist sich der teils unlösbaren Problematik dieses Themas durchaus bewusst, hätte sich jedoch zumindest eine Auseinandersetzung mit dieser nicht unerheblichen Thematik, bestenfalls sogar in einem eigenen Kapitel, gewünscht.

Kapitel I: Ägyptische Bronzefiguren: Material und Technik ist ein historischer Überblick über die Gewinnung, Herstellung und Verarbeitung verschiedener Kupfererze und Bronzelegierungen sowie den Herstellungsverfahren von Bronzestatuetten. Zwar bietet das Kapitel als solches gewiss eine sinnvolle Basis der weiteren Untersuchung. Jedoch fällt hier störend die sprunghafte Darstellungsweise auf, die teils sogar zu widersprüchlich scheinenden Angaben führt. Um diese Informationen wieder zu entwirren, 
sieht sich der Leser so mehrfach dazu gezwungen, zumindest die in den Fußnoten angegebene Literatur selbst noch einmal zu konsultieren, wenn nicht sogar darüber hinaus noch eigenständig weiter zu forschen, was sich als überaus zeitraubend erwiesen hat.

Zwei ausgewählte Beispiele mögen dies illustrieren:

1. Auf S. 6 erwähnt die Autorin zunächst Arsenbronzen, deren frühestes Aufkommen sie in das Alte Reich / Erste Zwischenzeit setzt, und spricht dann von den die Arsenbronzen allmählich ersetzenden Zinnbronzen. Ihre im nächsten Absatz gegebene Information zum Auftreten der Zinnbronze (ebenfalls) bereits im 3. Jt. v. Chr. ohne nähere Details stiftet Verwirrung und lässt den Leser ratlos über den von ihr insinuierten Prozess zurück. Die Konsultation der angegebenen Literatur ${ }^{3}$ und darüber hinaus ${ }^{4}$ führt dann zur Erkenntnis, dass Zinnbronzen durchaus schon seit der 2. Dynastie in Ägypten belegt sind, deren Verwendung in größerem Stil für uns bisher erst ab dem Mittleren Reich im materiellen Befund greifbar wird, wobei allerdings einschränkend bemerkt werden muss, dass zukünftige Materialanalysen diesen Zeitpunkt durchaus noch weiter nach hinten verschieben können ${ }^{5}$.

2. Ebenfalls auf S. 6 spricht die Autorin von neuen Forschungen zur Beherrschung der Bronzegusstechnik bereits im Alten Reich, um dann auf der nächsten Seite nur einige Zeilen weiter auf hohl gegossene Bronzestatuetten aus der Zeit Amenemhets III. zu verweisen, die als Beleg für „die Verwendung der Bronzegusstechniken schon im Mittleren Reich“ dienen. Wiederum gelangt man auch hier erst durch Nachschlagen in der angegeben Literatur ${ }^{6}$ zu der Erkenntnis, dass die Autorin einmal das Gießen im Vollgussverfahren (Beleg aus dem Alten Reich) und Hohlgussverfahren (Belege aus dem Mittleren Reich) meint.

Kapitel II: Kleidung, Kronen, Attribute: Dieser größtenteils rein deskriptive Abschnitt bietet eine methodisch ange-

3 D. Schorsch, The Manufacture of Metal Statuary: "Seeing the Workshop of the Temple", in: M. Hill, D. Schorsch (eds), Gifts for the gods: images from Egyptian temples. The Metropolitan Museum of Art, New York, 2007, 189-199.

4 A. J. Spencer, Catalogue of Egyptian Antiquities in the British Museum V: Early Dynastic Objects, London, 1980, 83-88.

5 Vgl auch A. Lucas, Ancient Egyptian materials and industries ${ }^{4}$, London, 1962, 219; P. T. Nicholson, I. Shaw (eds), Ancient Egyptian materials and technology. Cambridge, 2000, 152-154.

6 M. Hill, Charting Metal Statuary: The Archaic Period through the Pre-Thutmoside New Kingdom (ca. 3100-1479 B.C.), in: M. Hill, D. Schorsch (eds), Gifts for the gods: images from Egyptian temples. The Metropolitan Museum of Art, New York, 2007, 16; 201.4. legte sinnvolle Übersicht und Beschreibung über die dann im Folgenden häufig besprochenen ikonographischen Elemente der Götter- und Tierbronzen, wozu sie auch alle auftretenden Bekleidungselemente und Dazugehöriges wie Textilien und Perücken zählt.

Rezensentin hat hierzu jedoch Folgendes zu bemerken:

1. Auf S. 21-22 möchte die Autorin das Mittelteil des šnd. $w t$-Schurzes über das $b s 3 w$-Perlengehänge kurzerhand vom šsm.t-Gürtel ableiten. Dies ist so ohne Weiteres keineswegs möglich: zum einen gibt es keinen Hinweis darauf, dass das gefältelte Mittelteil des šnd. $w t$-Schurzes eine Nachahmung eines Perlengehänges sein könnte ${ }^{7}$, zum anderen ist der šsm.t-Gürtel ganz eindeutig ein die ganze Taille umspannender und über dem Schurz getragenes Accessoire, das nicht nur die Mitte des Schurzes bedeckt. ${ }^{8}$

2. Das Unterkapitel 2.2.2.2 Kronen, Kopftücher und Symbole dieses Abschnittes ist alphabetisch geordnet; von einer typologischen Ordnung oder Systematisierung wird ausdrücklich Abstand genommen, was Rezensentin für eine unglückliche Lösung hält, da so zusammengehörige Typen (z. B. Doppelkrone und ihre Einzelelemente Rote und Weiße Krone) auseinandergerissen werden, was $\mathrm{zu}$ vielen unnötigen Wiederholungen führt und die Herstellung von Bezügen erschwert. Auch sonst ist der oft unpräzise und sehr redundante Stil in diesem Abschnitt zu bemängeln. Im Haupttext Geschriebenes wird zum Teil noch einmal ausführlich in Fußnoten ausgebreitet (Vgl. S. 46, Anm. 182). Das Gleiche gilt übrigens für die alphabetische Abhandlung der Attribute in Kap. 3.4, $53 \mathrm{ff}$.

Kapitel III: Typologie beginnt mit einem knappen Abschnitt, in dem die Entscheidungskriterien der Autorin, Objekte in eine bestimmte typologische Gruppe einzuordnen, dargelegt werden. Ansonsten ist dem Konzept des Typus und der Zielsetzung der Typologisierung keine besondere Aufmerksamkeit gewidmet worden, was bei der von ihr bearbeiteten Objektgruppe aber durchaus interessant gewesen wäre. ${ }^{9}$ Auch die Problematik der in einigen

7 Vgl. G. Vogelsang-Eastwood, Pharaonic Egyptian Clothing, Studies in Textile and Costume History, 2, Leiden, 1993, 32 ff., die dieses Mittelteil als eigenständiges, aus Stoff (!) bestehendes Kleidungsstück identifiziert.

8 Vgl. P. E. Newberry, "SSMT", in: Studies presented to F. Ll. Griffith. London 1932, 316-323; K. H. Priese (Hrsg.), Staatliche Museen zu Berlin, Ägyptisches Museum und Papyrussammlung, 41.

9 W. Y. Adam, Archaeological Typology and Practical reality, Cambridge 1991. 
Fällen schwer umsetzbaren Trennung zwischen Typologie und Ikonographie, die sich oftmals überschneiden, so z. B. sichtbar bei der bei der Wahl eines eigentlich ikonographischen Elementes als Merkmal einer typologischen Gruppe (z. B. unterschiedliche Kronen, die derselbe Typus trägt) wird nicht angesprochen. Der Einleitung folgt eine umfangreiche Tabelle einer detaillierten und gut nachvollziehbaren Typologie der bearbeiteten Bronzestatuetten, an die sich Beschreibung, Deutung und eine „Lokale Spezifikation“ benannte kulttopographisch orientierter Kontextualisierung der einzelnen Statuettentypen anschließen. Dieser Abschnitt macht den Löwenanteil der Publikation aus und wird von dem im 2. Band niedergelegten umfangreichen Katalog und Abbildungen begleitet.

Allerdings begegnet hier zum ersten von vielen Malen das Problem der oftmals ungesicherten Herkunft. In einer Vielzahl von Fällen wäre hier seitens der Autorin etwas größere Vorsicht geboten gewesen, wie das Folgende demonstrieren soll:

Für den Großteil der mit der Herkunftsangabe „Bubastis" versehenen Statuetten verweist die Autorin auf den Katalog von Hilton Price. ${ }^{10}$ Diese Information muss aber keinesfalls zwangsläufig mit dem tatsächlichen Fundort übereinstimmen, denn die Antikenhändler von Zagazig, der modernen Stadt nahe der antiken Stätte, boten Funde aus der ganzen Umgebung an. So wird dann oft kurzerhand aus einem Ankauf in Zagazig ein Fund aus Bubastis. Demnach ist Bubastis lediglich als sicherer Erwerbungsort, aber nicht als sicherer Fundort anzusehen, die Stücke können auch durchaus aus anderen antiken Plätzen des Deltas stammen, je nach Aktionsradius des jeweiligen Händlers. So zum Beispiel bei der Statuette des Imhotep, Kat. Nr. 20 (Bd. II, 523): Als Herkunftsort ist bei der Autorin „Bubastis“ angegeben. Geht man aber der angegebenen Literatur zu diesem Stück nach, in diesem Falle dem $\mathrm{Mu}$ seumskatalog Antwerpen ${ }^{11}$, findet sich dort aber als korrektere Herkunftsangabe „Zagazig (Delta)“. Dieses Stück ist also als Beleg für einen Kult des Imhotep in Bubastis (s.u.) nicht mehr brauchbar.

Das Beispiel zeigt, dass die Arbeit von einem solche allseits bekannten Tücken von Herkunftsangaben thematisierenden Kapitel sehr hätte profitieren können. Natürlich würde ein solches die Schwierigkeiten nicht auflösen, jedoch in den fraglichen Fällen letztendlich eine differenziertere Analyse und Rekontextualiserung erlauben, bzw. deren Grenzen aufzeigen.

10 H. Price, A Catalogue of the Egyptian Antiquities in the Possession of F. G. Hilton Price, London, 1897.

11 Anonymous (ed.). Egypte onomwonden: Egyptische oudheden van museum Vleeshuis. Antwerpen: Stad Antwerpen, 1995.
Die Ausführungen der Verfasserin zu einer ThotBronze aus Bubastis (Typ 17, S. 94, Herkunftsangabe wiederum nach Hilton Price, s. Bd. II, 542) verdeutlicht dies noch einmal, legt aber auch weitere Schwierigkeiten der Arbeit offen: Da nach Ansicht der Autorin kein „Kultkontext" für Thot in Bubastis existiert, erwägt sie, die Statuette als Amulett zu deuten. Hier ist einerseits anzumerken, dass ein Filialkult für Thot in Bubastis keineswegs auszuschließen ist, sondern sich u. a. möglicherweise in Gestalt des bei Herodot, Hist. II, 138 erwähnten Tempel des „Hermes" und den dort aufgefundenen Dedikationsinschriften Osorkon I., die Thot durchaus prominent erwähnen ${ }^{12}$, nachweisen ließe. Schwerwiegender ist jedoch das Problem des ständigen, hier ablesbaren Zwanges, immer und ständig aus jeder Statuette gleich einen Lokalkult (re-) konstruieren zu müssen, dem sich offenbar auch die Autorin ausgesetzt fühlte.

Es ist doch vielmehr generell $\mathrm{zu}$ fragen, inwieweit solche Bronzefiguren, selbst bei gesicherter Herkunft, immer als Reflexion auf und Ausfluss eines etablierten Tempelkultes verstanden werden müssen. Auch wenn das Bemühen der Autorin in dieser Richtung überaus verständlich ist, da sie wie alle historisch arbeitenden Wissenschaftler letztendlich um die Entwicklung einer kohärenten Erklärung und Kontextualisierung, einer alles erklärenden story ihrer Objekte bemüht sein muss, betritt sie hier jedoch unsicheres Gelände. Deshalb wäre es lohnenswert gewesen, dies zuvor auch einmal theoretischmethodisch auszuleuchten. ${ }^{13}$ Für die Arbeit hätte dies insofern einen hohen Mehrwert besessen, da Fragen nach kultischer bzw. alltagsweltlicher Kontextualisierung der Bronzen, somit im engeren und weiteren Sinne Provenienzfragen, natürlicherweise im Zentrum ihrer Auswertungen stehen. Besonders die Frage nach der tatsächlichen Bezugnahme solcher Götterfigürchen auf lokale, nach unserem Verständnis durch monumentale Architek-

12 E. Naville, Bubastis, EEF 8, 1891, 60-62. Da die erhaltenen Reliefs in prominenter Stellung Atum zeigen, hat Labib Habachi den Tempel auch diesem Gott zugeschrieben, worin ihm seitdem andere Autoren gefolgt sind. Dies ist jedoch keinesfalls als sicher anzusehen: L. Habachi, Tell Basta, Supplément ASAE 22, Le Caire: Imprimerie de l'Institut français d'archéologie orientale 1957, 119-120; K. Kitchen, The Third Intermediate Period in Egypt (1100-650 B.C.) $)^{2}$, Warminster, 1996, 304; K. Jansen-Winkeln, Inschriften der Spätzeit, Teil II: die 22.-24. Dynastie, Wiesbaden, 2007, 38; R. Ritner The Libyan anarchy: inscriptions from Egypt's Third Intermediate Period. Writings from the Ancient World 21, Atlanta; Leiden: Society of Biblical Literature; 2009, 249.

$13 \mathrm{Zu}$ dieser Problematik s. K. Jansen-Winkeln, Die Rolle des Unbekannten in der ägyptischen Geschichte, in: M. Fitzenreiter (Hrsg.), Das Ereignis: Geschichtsschreibung zwischen Vorfall und Befund. Workshop vom 03.10. bis 05.10.08. London, 155-161. 
tur und organisierte Kultvorgänge „offiziell“ fixierte Kulte, ist bei genauerer Betrachtung noch immer ungenügend geklärt.

Einige Detailbeobachtungen zu diesem Kapitel:

1. S. 90: Bei der Interpretation ihres Typus 13 führt die Autorin aus, dass die Darstellung des Anubis mit einer runden Mähne (anstatt einer dreiteiligen Strähnenperücke) möglicherweise als Anspielung auf seine Verbindung $\mathrm{zu}$ felidengestaltigen Göttinnen wie Bastet (Löwengöttin $\rightarrow$ Mähne) zu verstehen sei. Jedoch verfügt der in Ägypten vertretene Goldschakal (Canis aureus) durchaus über eine kleine Halsmähne, die Darstellung könnte also eher naturalistisch am lebenden Vorbild orientiert sein; Gleiches gilt für den afrikanischen Goldwolf (Canis anthus).

2. S. 106: Nefertem: Die in Fußnote 149 genannten Katalognummern tragen keine Inschrift, können also auch nicht als Belege für beschriftete Statuetten, die Nefertem als Sohn der Sachmet oder Bastet nennen, angeführt werden.

3. S. 109-113: Hier wird jeweils beim Stichwort „Lokale Spezifikation“ für die verschiedenen Osiris-Jah-Formen immer wieder derselbe Inhalt wiederholt, da es sich ausschließlich um das Serapeion als Verehrungsort des Osiris-Jah und damit als Mondkultzentrum, und Verbindungen zwischen Osiris, Apis und Mond handelt. Dies hätte man besser zusammenfassend für alle dieser Formen einmal in methodischer Weise abhandeln können.

4. Auf S. 126-157 behandelt die Autorin die Vielzahl der belegten Typen von Kindgöttern. Auch hier wird immer wieder auf die generelle Wichtigkeit ägyptischer Kindgötter sowie die Schwierigkeit ihrer konkreten Identifikation hingewiesen. Dies hätte, ebenso wie andere Themen, die zentrale Punkte der Arbeit berühren und häufig vorkommen, durch ein einleitendes methodisches Kapitel zum Stand der Forschung und den Grundlinien der Kindgottverehrung methodisch straffer organisiert werden können.

Das Kapitel endet mit einem Fazit (Kap. III.8, S. 374-380) zur beobachteten Typologie des Korpus, das immerhin als Ergebnis festhalten kann, dass keine absolut eindeutige Identität von Typus und Gottheit gegeben ist, sondern eine bestimmte Gottheit durch verschiedene Typen verkörpert werden kann, wobei gewisse ikonographische Merkmale oft eine größere Rolle spielen als die Grundgestalt selbst. Auch die archäologischen Hinweise auf die ehemalige Aufstellung vieler Bronzen in Schreinen und ihre Kombination zu Gruppen findet hier erstmals Erwähnung.
Kapitel IV: Herkunft der Bronzestatuetten widmet sich in mehreren Unterkapiteln der Besprechung der zuvor konstatierten Fundorte. Hierunter befinden sich wichtige archäologische Stätten des Nildeltas, auf deren Lokalgötter und -kulte die Autorin fokussiert. So nützlich im Einzelnen diese Aufstellung ist, so sehr fehlt auch hier wieder ein methodischer Zugang. Neben der wiederum rein alphabetischen Abhandlung der übrigens besser geographisch anzuordnenden Fundorte hätte eine übersichtliche exemplarische Gegenüberstellung der jeweils am Fundort auftretenden Bronzetypen mit den davon unabhängig belegten Lokalgöttern und -kulten viel mehr Klarheit schaffen können. Hierbei hätte wesentlich deutlicher herausgestellt werden können, was der Leser bei der Durchsicht der einzelnen Typtabellen zu den jeweiligen Fund-bzw. Herkunftsorten nicht umhin kommt, festzustellen: dass nämlich die Bronzen meist gar nicht besonders auffällig die Lokalkulte abbilden, jedenfalls weit weniger, als Autorin dies zu sehen meint. Es entsteht immer mehr der Eindruck, die Autorin habe hier etwas hineingelesen, was sie bereits vorher erwartete. Als Beispiel seien die Funde aus Bubastis und Memphis angeführt:

1. Bubastis: Bei der Durchsicht der bei Weiß, 387-389 publizierten Tabelle der verschiedenen Statuettentypen mit der Fundortangabe Bubastis ergibt sich Folgendes: Den größten Anteil mit 43 Objekten stellen zwar erwartungsgemäß Bronzestatuetten und Köpfe von Katzen als Tiere, die damit knappe $26 \%$ der Gesamtmenge ausmachen. Diese stammen jedoch, soweit die Zuschreibung an den Fundort nicht ohnehin zweifelhaft ist und einfach später aufgrund des Typus erfolgte (s. o.), vermutlich hauptsächlich aus der Katzennekropole. Diese Tiernekropole ist aber nicht ohne Weiteres den im Tempelbereich verorteten Kulten zuzuordnen, sondern davon getrennt zu betrachten, wie die Autorin selbst später ausführt (s.u.). Die Bronzen, die die Göttin Bastet (anthrophomorph mit Katzenkopf) abbilden, immerhin die Hauptgöttin des Ortes, stellen mit nur fünf Objekten lediglich überraschende $3 \%$ der Gesamtmenge, die Darstellungen einer Löwengöttin (ohne nähere Zuweisbarkeit an Bastet oder Sachmet) einschließlich der Kragenprotome zwölf Objekte, d. h. 7,2\% der Gesamtmenge der ausgewerteten Objekte. Die Darstellungen des Osiris nun sind sogar zahlreicher, mit 13 Objekten machen sie 7,8 \% der Gesamtmenge aus. Da ein spezieller Kult des Osiris in Bubastis ansonsten nicht belegt ist, ließe sich spekulieren, ob diese Figuren nicht ebenfalls aus der Katzennekropole stammen (was Autorin auch tut: S. 389-390). Jedoch wäre die Herkunft aus den um- 
fangreichen Stadtnekroplen von Bubastis ebenso erwägenswert.

Der neben Bastet zur Triade von Bubastis gehörende Sohn der Göttin, Nefertem, ist dagegen nur mit fünf Bronzen (ebenfalls nur etwa $3 \%$ ) vertreten, obwohl er einer der Hauptgötter des Ortes war und nach ihren Erwartungen demnach stärker vertreten sein müsste. Quantitativ identisch sind nun Bronzen des Anubis, der unabhängig davon in Bubastis nicht mit einem speziellen Filialkult oder dergleichen belegt ist. Dasselbe gilt für Kindgötterbronzen, die man ebenfalls nicht in hoher Zahl erwartet hätte; sie sind aber mit neun Objekten (5,4 \%) fast so häufig wie die der Bastet und des Nefertem zusammengenommen. Bronzen des Mahes (Miysis), eines weiteren Sohnes der Bastet in der Götterkonstellation von Bubastis, gibt es dagegen nur dreimal (1,8\%), wohingegen Neith und Apis, die man schwerlich mit speziellen Lokalkulten in Bubastis in Verbindung bringen kann, ebenfalls auf je zwei Bronzen kommen, also nur unwesentlich weniger. Imhotep wiederum, für den die Autorin einen bedeutenden Kult in Bubastis ansetzt (S. 78, 389, ohne allerdings die relevante Literatur dazu zu nennen ${ }^{14}$ ), bringt es auf ebenso viele bzw. ebenso wenige Statuetten (zwei). Es wird schon aus dieser noch völlig oberflächlichen Analyse klar, dass sich die Lokalkulte offenbar doch nicht so eindeutig im Befund abbilden, wie die Autorin dies darstellen möchte, zumal wenn man die Katzenbronzen aus den Nekropolen, die ja nicht einfach mit den Lokalkulten gleichzusetzen sind, abzieht. Nüchtern betrachtet könnte das Typenspektrum der Bronzestatuetten auf fast jeden anderen beliebigen Fundort in Ägypten hinweisen. Hinzu kommt, dass sogar davon auszugehen ist, dass eine unbekannte Anzahl der oben in die Zählung hineingenommenen Katzenbronzen bzw. auch der Bronzen der Göttin Bastet ihre Fundortzuweisung weniger der tatsächlich festgehaltenen Auffindung sondern ihrem Charakter verdankt, mit anderen Worten: eine Katzenoder Bastetbronze unbekannter Herkunft wurde im Antikenmarkt automatisch mit dem Label „Bubastis“ versehen, ohne dass dies immer, wie oben ausgeführt, den Tatsachen entsprechen muss ${ }^{15}$.

14 L. Habachi, P. Ghalioungui, The House of Life“ of Bubastis, CdE 46, 1971, 59-71.

15 Vgl. auch E. Naville, Bubastis, EEF 8, 1891, 53, der berichtet, dass bei seinen Grabungen in der Katzennekropole die von ihm untersuchten noch nicht gestörten Gruben keineswegs eine große Menge von Bronzen gefunden wurde.
2. Zum Vergleich nun noch ein Blick auf den Typenbefund aus Memphis: Hier machen nicht etwa die bekannten großen Lokalgötter wie Ptah, Sachmet Nefertem und Bastet die Hauptmenge aus, sondern Kindgötterbronzen, nämlich mit 27 Objekten 15,6\%, gefolgt von Apisbronzen (24 Objekte, 13,9\%) und Osirisbronzen (15 Objekte, also 8,7 \%). Ptah ist dagegen lediglich mit acht Statuetten vertreten (4,6\%), Bastet mit sechs (3,5\%), Nefertem mit zwei (1,2 \%!) und Sachmet mit nur einer einzigen Bronze (0,58 \%!). Lediglich die Anzahl der Apisbronzen entspricht den Erwartungen. Bestätigung findet dies bei einem Blick auf die Befunde von Mendes und Sais: unter den (zugegebenermaßen wenigen) Bronzen von Mendes findet sich keine einzige Darstellung des Ba von Mendes. Die zahlreichen Bronzen aus Sais wiederum bieten nur sechs Statuetten der Neith, dafür aber neun Bronzen von Löwengöttinnen (Wadjet, Mut) 14 Kindgötterstatuetten, usw., usf.

Diese Analysen ließen sich noch wesentlich detaillierter fortsetzen, jedoch ist dies nicht Aufgabe der Rezensentin. Es sollte lediglich aufgezeigt werden, dass sich dem Betrachter letztlich ein stark durchmischter Befund darbietet, wie es die Autorin z. B. für den „heterogenen“ Befund von Giza feststellt (S. 405). Hier wäre der Frage nachzugehen gewesen, inwieweit dies entweder auf eine nicht unerhebliche Anzahl von fehlerhaften Fundortzuschreibungen zurückzuführen ist oder die Lokalkulte sich eben doch nicht 1:1 im Befund reflektieren, und welche Gründe dies hat. Vor allem Letzteres hätte nach Ansicht der Rezensentin ein Kernthema der Arbeit bilden müssen. ${ }^{16}$

Tatsächlich gelangt die Autorin selbst viel später in Kapitel V auf S. 476 und dann besonders S. 479, Anmerkung 2 und S. 480, Anmerkung 4 bei ihrer Abhandlung zu den Tierkulten zu der mit dem oben umrissenen Problem verbundenen Erkenntnis, dass nicht jede Tierbronze aus den Tiernekropolen eine Entsprechung in einem lokalen Tempel der damit verbundenen Gottheit haben muss, bzw. Tiere unterschiedliche Götter oder unterschiedliche Aspekte einer Gottheit repräsentieren können. Diese Erkenntnis hätte jedoch schon in den Auswertungskapiteln $\mathrm{zu}$ den einzelnen Fundorten Eingang und Anwendung finden sollen. So jedoch stehen diese Kapitel etwas zusammenhanglos da.

$16 \mathrm{Zu}$ diesem hochinteressanten Problemkreis hätte sich die Autorin beispielsweise auf Vorarbeiten wie die von P. Kopp, D. Raue, Reinheit, Verborgenheit, Wirksamkeit. Innen-, An- und Außensichten eines ägyptischen Sanktuars jenseits der zentralen Residenzkulte, Archiv für Religionsgeschichte 10, 2008, 44 ff. stützen können. 
Dementsprechend aufschlussreicher sind da doch die Bronzefunde aus Sakkara, die vor allem aufgrund der besser dokumentierten Herkunft, namentlich aus zwei Depositen, verlässlichere Anhaltspunkte $\mathrm{zu}$ offerieren scheinen. Die Autorin versucht dann auch, die Bronzefunde aus Sakkara mit denen aus Memphis thematisch zu verbinden, um somit dem doch recht disparaten Befund aus Memphis im Hinblick auf die erwünschte Korrelation (anderweitig) belegter Lokalgottheiten etwas auf die Beine $\mathrm{zu}$ helfen. Dabei muss sie etwas diffus bleiben und z.Bsp. dem in Sakkara (sechs Statuetten) zahlenmäßig fast ebenso häufig wie in Memphis auftretenden Ptah dann generell der „Bedeutungssphäre Memphis“ (S. 418) zuweisen, wobei seine Beziehung zu Sakkara konkret dann aber wiederum unbeleuchtet bleibt. All dies ist methodisch doch recht unbefriedigend, hier wäre ein differenzierteres Vorgehen wünschenswert gewesen. Vor allem fehlt eine vorherige Auseinandersetzung mit dem Begriff und dem Phänomen der Lokalkulte, der genau hieran, an solchen Befunden, hätte erprobt werden müssen.

Ohnehin kann die Rezensentin höchstens bei den Funden aus den Tiernekropolen, vor allem in Sakkara eine gewisse Korrelation zwischen vorher bekannten kultischen Gewichtungen und dem dort bisher nachgewiesenen Typenspektrum der Bronzestatuetten nachvollziehen (vor allem Serapeion und Falkengalerien, s. dazu aber auch unten). Ob dies auf das einer Stadt gegenüber enger fassbare Funktionsspektrum einer Nekropole gründen könnte, ja, in welchem Umfang Fundorte Funde überhaupt determinieren, hätte sich gelohnt, einmal näher zu erörtern. Fazit ist die auf S. 460 in der Zusammenfassung zu Kap. IV getroffene Aussage: „Eine wichtige Feststellung ist, dass das Typenspektrum der Bronzestatuen in der Regel die vorhandenen Kulte widerspiegelt. Die Weihegaben werden also gezielt dem lokalen Kult angepasst.“ Das muss Rezensentin aber deutlich ablehnen. Jedoch ist der Autorin bezüglich der Aufstellungspraxis zuzustimmen, wenn es dort heißt: „Tierbronzen werden grundsätzlich in Tiernekropolen und deren Bereiche geweiht und nicht in Tempel (...)“ (S. 461).

Kapitel V: Die Funktion der Bronzefiguren beginnt mit einem sehr nützlichen Exkurs zum Begriff des Votivs, der erst einmal prinzipiell die Unterschiede zwischen Opfer und Votiv aufzeigt. In Kapitel V.1 werden ebenfalls sehr sinnvoll noch einmal die Fundkontexte der behandelten Bronzefiguren näher diskutiert, auch wenn dieses Kapitel möglicherweise besser dem Kapitel zur Herkunft der Bronzestatuetten vorgeschaltet werden können, da hier erstmals die dort immer wieder nur angerissene Thematik der Unterscheidung zwischen Tempel und Tiernekropole als Fundort bzw. auch ursprünglicher Aufstellungsort nun einmal methodischer ausgeleuchtet wird. Auch gemessen daran, dass hier ebenfalls die Cachettes, die die verlässlichsten Anhaltspunkte hinsichtlich der Provenienz der Statuetten des Corpus bilden, behandelt werden, ist dieses Kapitel nach Ansicht der Rezensentin zu knapp geraten.

In Kap. V. 2 Tierbronze vs. Götterbronze und der Anlass der Weihung nähert die Autorin sich nun tiefergehend den in Kapitel IV bereits mehrfach erwähnten Tiernekropolen und der damit verbundenen Problematik des ägyptischen Tierkultes. Bei der Darstellung orientiert sich Weiß hauptsächlich an den Thesen Dieter Kesslers, der dazu bekanntermaßen umfangreich gearbeitet hat, und verbindet dies mit nützlichen Hinweisen auf die Thematik der Tierbronzen, wobei man sich jedoch auch hier eine detailliertere statistische Auswertung gewünscht hätte. Einen wichtigen, bei Kessler bereits diskutierten Aspekt übersieht sie hierbei, nämlich das Missverhältnis zwischen den in Sakkara Nord aufgefundenen Bronzen insgesamt und dem Anteil der Bronzen an dieser Gesamtmasse, die tatsächlich die dort bestatteten Tiere abbilden, was sich mit den oben ausgeführten Zweifeln der Rezensentin bezüglich der Reflexion der jeweiligen Lokalkulte im Corpus der Statuetten trifft. ${ }^{17}$

Mit der Ablehnung der veralteten Vorstellung der Bronzen, besonders der Tierbronzen als Ausdruck persönlicher Frömmigkeit und individueller Weihegaben von Pilgern folgt sie, sicher zu recht, Dieter Kessler. Jedoch ist auch die Imagination einer vollkommen entpersonalisierten, „staatlich“ kontrollierten Weihetätigkeit „standardisierter" Votivgaben (S. 478) und Votivgabenprozesse, die mehr oder weniger ausschließlich im Rahmen von Festen stattfinden, nach Ansicht der Rezensentin nicht ganz problemlos. Richtig benennt die Autorin den schon von Kessler erarbeiteten Zusammenhang zwischen lokaler Tiernekropole und lokalen Götterfesten, an denen heilige Tiere teilhatten; hier verweist sie zwar auf Bubastis, jedoch fehlt wiederum eine differenziertere Auseinandersetzung. So bietet, um nur ein Beispiel zu nennen, pBrooklyn 47.218.84,IX.6-IX. $8^{18}$ Belege für einen lokalen Mythos vom Sieg der Bastet über Seth, der sicher mit dem von Herodot (Hist. II, 59-60) berichteten und auch schon früher belegten Fest der Bastet, das als Triumphfahrt der Göttin auf ihrem Ischeru begangen wurde, zu verbinden ist.

17 D. Kessler, Die heiligen Tiere und der König. Teil I: Beiträge zu Organisation, Kult und Theologie der spätzeitlichen Tierfriedhöfe. ÄAT 16. Wiesbaden 148.

18 D. Meeks, Mythes et légendes du Delta d'après le papyrus Brooklyn 47.218.84. MIFAO 125, Le Caire: Institut Français d'Archéologie Orientale, 2006, 19-25. 
Die Autorin kommt zu dem Schluss, dass die Tierbronzen in ihrem Gesamtcorpus mit dem (offiziellen) Kult der in den Tiernekropolen bestatteten heiligen Tiere und dem Königskult in Zusammenhang stehen und dabei sowohl regionale als auch überregionale religiöse Aspekte abdecken. Den stark osirianischen Charakter dieser Anlagen sieht sie durch die auch in den Tiernekropolen sehr häufig auftretenden Osirisbronzen bestätigt, worin die Rezensentin ihr unbedingt zustimmt (s. z. B. Sakkara Nord, Falkengalerien. S. 433-436). Während damit in den Tiernekropolen sowohl Tier- als auch Götterbronzen geweiht werden können, sind Tierbronzenvotive in den Lokaltempeln dagegen ungebräuchlich. Hier kommen vielmehr anthropomorphe bzw. mischgestaltige Götterabbildungen zum Einsatz (S. 480). Diese Feststellung ist keineswegs banal, sondern für zukünftige

(Re)kontextualiserungen äußerst wichtig und bietet einen sehr nützlichen Ansatz für weitere Forschungen zum Phänomen der Lokalkulte.

Der bereits in Kap. V.2 mehrfach angesprochenen Thematik der Identität der Stifter ist dann dankenswerterweise Kapitel V.3 Stifter und Weihinschriften nochmals gewidmet. Da die Verfasserin hier stark auf den epigraphischen Befund angewiesen ist, der sich naturgemäß recht übersichtlich gestaltet, kann sie nicht zu allzu tiefgehenden Ergebnissen kommen, jedoch immerhin herausarbeiten, dass die Weihenden, wenn eine Titulatur angegeben ist, sehr häufig Priestertitel tragen, die allerdings nur zum Teil mit dem Votiv der dargestellten Gottheit korrelieren (S. 485). Priester treten auch als Vermittler der Stiftung auf, wobei die Stellung des Stifters unklar bleibt. ${ }^{19}$ Obwohl die Autorin eine allgemeine Schematik der Weihinschriften gibt (S. 483), vermisst die Rezensentin doch neben der simplen Wiedergabe im Katalog (Band 2), eine systematische Auflistung und ausführliche Besprechung der zur Verfügung stehenden Inschriftenbelege und dazugehöriger Vergleichsbeispiele, wie sie der Wichtigkeit dieser Quellengruppe angemessen gewesen wäre.

Kapitel V.4 Die Zeitstellung der Bronzestatuetten wäre möglicherweise ebenfalls zu Beginn der Monographie besser aufgehoben gewesen. Weiß bespricht hier generell die Chronologie der Bronzestatuettenproduktion in Ägypten, die spezielle Problematik der Datierung von Bronzestatuetten sowie einige sicher zu datierende Fundkontexte.

19 Vgl. auch F. Colin, Les Fondateurs du Sanctuaire d'Amon à Siwa, in: W. Clarysse, A. Schoors, H. Willems (eds.), Egyptian Religion: the Last Thousand Years. Studies dedicated to the memory of Jan Quaegebeur: part I. Orientalia Lovaniensia Analecta 84. Leuven, 1998, 346349.
Allerdings sind dabei kaum Verweise auf ihren eigenen Objektkatalog zu finden, und das Kapitel verbindet sich dadurch nicht konkret mit ihrer Arbeit, obwohl gerade hier viele Unsicherheiten der Datierung bestehen, die hätten thematisiert werden müssen.

Kapitel V.5 Zusammenfassung bildet den Abschluss des eigentlich ägyptologischen Teils der Arbeit. Diese ist recht knapp gehalten und betont noch einmal die große Rolle der ab der Dritten Zwischenzeit immer stärker im Befund auftretenden Bronzestatuetten als „Ausdrucksform religiöser Thematik" (S. 491) vermutlich besonders im Rahmen großer Götterfeste, worin der Autorin zweifelsfrei zuzustimmen ist. Inwieweit sich hier tatsächlich eine „enge Bindung“ des Stifters an den jeweiligen Tempel und Kult ergab und wie man sich diese konkret vorzustellen hätte, wäre vielleicht noch $\mathrm{zu}$ diskutieren. Nützlich sind die Hinweise auf die Herkunft des Materials ${ }^{20}$ und die Anfertigung in den Tempelwerkstätten, störend wirkt hier jedoch wie auch sonst der unreflektiert gebrauchte Terminus „staatliche Seite“, der nirgendwo deutlich erklärt wird.

Kapitel VI: Bronzen innerhalb des kulturellen Austauschs behandelt zunächst unter VI.1. Bronzefunde ägyptischer Statuetten außerhalb Ägyptens. Hier nimmt sich die Autorin speziell der Funde aus Askalon und der griechischen Welt an, wobei das Heraion von Samos aufgrund der großen Menge an Objekten besonders hervorsticht. Dieses interessante Kapitel, das einem Exkurs entspricht, verdankt sich offenbar der Mitwirkung der Autorin am SFB 295 "Kulturelle und sprachliche Kontakte" an der Johannes Gutenberg-Universität Mainz (s. S. xxxiii, Vorwort). Unter V.1.2 Kommentar (zu den außerhalb Ägyptens gefundenen ägyptischen Bronzen) findet sich dann eine Abhandlung zu den ägyptischen Götter- und Lokalkulten, die man besser und vertiefender in einem eigenen Kapitel bereits zu Beginn untergebracht hätte, da sie Kernfragen der gesamten Arbeit berühren.

Im Endergebnis kommt die Autorin zu dem Schluss, dass es im Gegensatz zu den Ansichten früherer Autoren keine ägyptischen Kulte im Heraion von Samos gegeben habe, da sich die dort aufgefundenen ägyptischen Bronzen auch ohne große Schwierigkeiten im Licht des Herakultes und ihrer zahlreichen Aspekte deuten ließen (S. 504-506). Ein weiteres bemerkenswertes Ergebnis scheint zu sein, dass das Typenspektrum der Bronzefiguren aus Samos Bezüge zum memphitischen Raum aufweist (S. 509), den sie als hauptsächlichen Begegnungsraum von Ausländern

20 Wobei aber auch ein privater, durch Tauschhandel erworbener Besitz kleiner Metallmengen nicht auszuschließen ist. 
mit ägyptischen Kulten ansehen möchte. Auch wenn Memphis hierfür natürlich sehr gute Voraussetzungen bietet, würde die Rezensentin solche Schlussfolgerungen mit Blick auf die oben ausführlich besprochene Problematik der fehlenden eindeutigen Korrelationen zwischen Lokalkulten und Bronzetypen vorsichtiger ziehen wollen. Schließlich kann die Autorin generell auf die durch Inschriften belegte Weihetätigkeit von Tierstatuetten durch Ausländer in Ägypten besonders im Rahmen von Neujahrsfesten verweisen, die sie bereits in Kapitel V.2 und V.3 ausführlicher diskutiert hat.

Die besprochene Monographie zu den Götter- und Tierbronzen Unterägyptens ist generell schon insofern positiv zu bewerten, als sich Katja Weiß hiermit eines vernachlässigten, spröden und disparaten Themas ange- nommen hat. Der Katalog insbesondere bietet mit den zahlreichen gut erschlossenen Typen und dem ausführlichen Abbildungsteil ein hervorragendes Nachschlagewerk. In der systematischen Katalogisierung und Präsentation der über viele Sammlungen verstreuten Bronzefiguren besteht dann auch die hauptsächliche Stärke dieser Arbeit; sie führt allerdings trotz einiger Ansätze nicht sehr weit darüber hinaus, da hier zu wenig methodisch und problemorientiert vorgegangen wurde. Die zugegebenermaßen äußerst schwierige Deutung und Rekontextualiserung der Funde, vor allem hinsichtlich des Themenkreises der miteinander verknüpften Lokalkulte, Tiernekropolen, Tierkulte und religiös motivierten Handlungen menschlicher Akteure wäre noch immer wesentlich $\mathrm{zu}$ leisten. 